\title{
Controlling Chaotic Behavior of Heavy to Light Hole Mixing Tunneling by External Electric Fields
}

\author{
Cheng Juang, Member, IEEE, J. Y. Wang, and Jonq Juang
}

\begin{abstract}
Oscillatory and chaotic motion of heavy to light hole mixing tunneling in asymmetric coupled quantum-well structures can be controlled by an external electric field. Chaotic behavior occurs if the heavy-hole state in the first well is aligned with the light-hole state in the second well under a significant in-plane vector $k_{\|}$. Oscillatory motion is recovered if the external electric field disrupts the alignment between the heavy-hole state in the first well and the light-hole state in the second well.
\end{abstract}

Index Terms - Controlling chaos, coupled quantum wells, heavy to light hole mixing tunneling, simplex projection method.

\section{INTRODUCTION}

$\mathbf{T}$ HE possibility of utilizing interwell coherent tunneling in coupled quantum wells to generate the oscillation signal has been proposed by Luryi [1]. Considerable efforts have been devoted to this oscillation effect due to its rich physical natures and possible device applications in the range of terahertz radiation [2]-[4]. For the valence band, in addition to the spatial hole tunneling (from one well to the other), mixing tunneling (between heavy hole $(\mathrm{HH})$ and light hole (LH) states) are also involved in the process due to the band mixing effects $\left(k_{\|} \neq 0\right)$ [5], [6]. The tunneling between heavy and light states has been verified using the timeresolved luminescence [7], [8]. Futhermore, the oscillatory motion via the heavy to light tunneling were also verified using the terahertz spectroscopy [9]. In theory, the oscillatory motion has been described by the time-dependent Schrödinger operator with the Luttinger Hamiltonian [6]. By calculating the tunneling probability for each case, the mixing tunneling and spatial tunneling which happen at the same time can be clearly resolved.

The interplay between tunneling and chaos in a coupled quantum well potential has become an interesting subject due to the nonlinear nature of tunneling effects [10], [11]. In this work, it is suggested that chaotic behavior in the valence band of the mixing tunneling could occur [12] and be controlled by an external electric field, as illustrated in Fig. 1. Two competing tunneling processes have contributed to the heavy to light hole mixing tunneling in coupled quantum wells: $\mathrm{HH}^{\alpha}$

Manuscript received February 4, 1997; revised March 31, 1997. This work was supported by the National Science Council of R.O.C. under Contract 862215-E159-001 and Contract 86-2115-M009-018.

C. Juang is with the Electronics Department, Ming Hsin Institute of Technology, Hsinfeng, Hsinchu, Taiwan 300, R.O.C.

J. Y. Wang and J. Juang are with the Department of Applied Mathematics, National Chiao Tung University, Hsinchu, Taiwan 300, R.O.C.

Publisher Item Identifier S 0018-9197(97)05430-4. to $\mathrm{LH}^{a}$ and $\mathrm{HH}^{a}$ to $\mathrm{LH}^{b}$, where the superscripts $a$ and $b$ denote the hole state in the first and the second well, respectively. $\mathrm{HH}^{a}$ to $\mathrm{LH}^{a}$ is due to the band mixing vector $k_{\|}$[9] while $\mathrm{HH}^{a}$ to $\mathrm{LH}^{b}$ is due to the spatial alignment of $\mathrm{HH}^{a}$ and $\mathrm{LH}^{b}$ states [5], [8]. When one process is dominant, oscillatory motion between the $\mathrm{HH}$ and $\mathrm{LH}$ states occurs. For example, a small in-plane vector $k_{\|}$and alignment of $\mathrm{HH}^{a}$ and $\mathrm{LH}^{b}$ results a dominant case of $\mathrm{HH}^{a}$ to $\mathrm{LH}^{b}$ tunneling. The other is that misalignment between $\mathrm{HH}^{a}$ and $\mathrm{LH}^{b}$ states results a dominant case of $\mathrm{HH}^{a}$ to $\mathrm{LH}^{a}$ tunneling. When these two processes are compatible, the oscillatory motion between $\mathrm{HH}$ and $\mathrm{LH}$ tunneling collapses. The tunneling probability in the oscillation collapse region can be shown to be chaotic using the simplex projection method [13]-[14].

In this nature, external electric fields can be used to switch between the oscillatory motion and chaotic motion. With a proper design of asymmetric coupled quantum-well (QW) structures, $\mathrm{HH}^{a}$ to $\mathrm{LH}^{a}$ and $\mathrm{HH}^{a}$ to $\mathrm{LH}^{b}$ are compatible, giving rise to a chaotic motion. Then, by adding an external electric field to the coupled well system, the $\mathrm{HH}^{a}$ and $\mathrm{LH}^{b}$ states become misaligned. Thus, $\mathrm{HH}^{a}$ to $\mathrm{LH}^{a}$ becomes dominant and oscillatory motion is back. The purpose of this paper is to show that chaotic behavior of the mixing tunneling can be controlled by the external electric fields. Section II describes the numerical techniques for the time-dependent Schrödinger operator and the simplex projection method. The effects of the external electric field on the wavefucntions and tunneling probabilities will be studied in Section III. Section IV examines the tunneling probabilities with and without electric fields using the simplex projection method to establish a chaotic or oscillatory motion of the particles.

\section{METHOD OF THE NUMERICAL ANALYSIS}

\section{A. The Time-Dependent Schrödinger Operator}

The time-dependent Schrödinger equation with the reduced $2 \times 2$ Luttinger Hamiltonian $H_{h}$ is written by [6], [15]

$$
\begin{aligned}
H_{h} & \left(\begin{array}{l}
\phi^{1}(x, t) \\
\phi^{2}(x, t)
\end{array}\right) \\
& =\left(\begin{array}{cc}
P+Q+V_{h}(z) & \tilde{R} \\
\tilde{R}^{*} & P-Q+V_{h}(z)
\end{array}\right)\left(\begin{array}{l}
\phi^{1}(x, t) \\
\phi^{2}(x, t)
\end{array}\right) \\
& =i \hbar \frac{\partial}{\partial t}\left(\begin{array}{c}
\phi^{1}(x, t) \\
\phi^{2}(x, t)
\end{array}\right)
\end{aligned}
$$




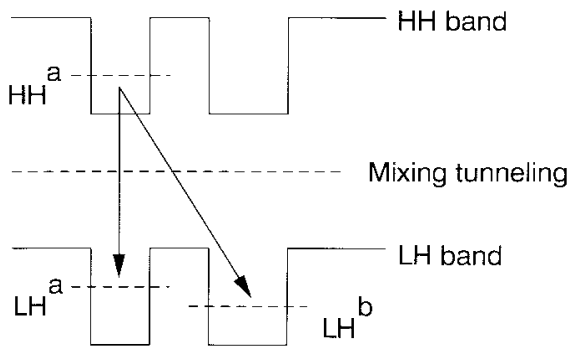

Fig. 1. Schematic potential profiles of the mixing tunneling in an asymmetric coupled quantum wells due to $\mathrm{HH}^{a}$ to $\mathrm{LH}^{a}$ and $\mathrm{HH}^{a}$ to $\mathrm{LH}^{b}$ tunneling.

where

$$
\begin{aligned}
& P=\frac{1}{2}\left(\frac{\hbar^{2}}{m_{0}}\right) \gamma_{1} k_{\|}^{2}-\frac{1}{2}\left(\frac{\hbar^{2}}{m_{0}}\right) \frac{\partial}{\partial z} \gamma_{1} \frac{\partial}{\partial z} \\
& Q=\frac{1}{2}\left(\frac{\hbar^{2}}{m_{0}}\right) \gamma_{2} k_{\|}^{2}+\left(\frac{\hbar^{2}}{m_{0}}\right) \frac{\partial}{\partial z} \gamma_{2} \frac{\partial}{\partial z} \\
& \tilde{R}=\left(\frac{\hbar^{2}}{m_{0}}\right)\left(\frac{\sqrt{3}}{4}\left(\gamma_{2}+\gamma_{3}\right) k_{\|}^{2}-\frac{\sqrt{3}}{2} k_{\|}\left(\gamma_{3} \frac{\partial}{\partial z}+\frac{\partial}{\partial z} \gamma_{3}\right)\right)
\end{aligned}
$$

and $V_{h}(z)$ is the potential profile of the coupled QW structures, $\phi^{1}(x, t)$ and $\phi^{2}(x, t)$ are $\mathrm{HH}$ and LH state envelop wave functions, respectively, $\gamma_{1}, \gamma_{2}$, and $\gamma_{3}$ are the Luttinger parameters and are position-dependent in the heterojunction structures, $m_{0}$ is the electron rest mass, and the in-plane vector $k_{\|}^{2}=k_{x}^{2}+k_{y}^{2}$. The external electric field $F$ is then added to the potential profile $V_{h}(z)$. The discretization of (1) with respect to time gives [6]

$$
\left(1+\frac{i \delta}{2 \hbar} H_{h}\right)\left(\begin{array}{c}
\phi_{n+1}^{1} \\
\phi_{n+1}^{2}
\end{array}\right)=\left(1-\frac{i \delta}{2 \hbar} H_{h}\right)\left(\begin{array}{c}
\phi_{n}^{1} \\
\phi_{n}^{2}
\end{array}\right)
$$

where $\delta$ and $n$ are the time spacing and time index, respectively. This discrete-time technique preserves normalization of the wave function and introduces no extra nonlinear effect to the system. This difference equation can be written in a linear $A x=b$ matrix equation with $A$ being a complex symmetry matrix. The matrix is then solved using the $\mathrm{L}-\mathrm{U}$ decomposition technique.

\section{B. The Simplex Projection Method}

To study the motion of a particle in a physical system, the simplex projection method is used [13]-[14]. This method uses a reconstructed trajectory vector to make short- and longterm predictions about future behaviors based on the previous observed information. The prediction is based on a piecewiselinear approximation of the past patterns in a time series. By comparing the predicted and actual trajectories, chaotic behavior can be established if the accuracy of the prediction falls off as the prediction time increases.

The algorithm is summarized as follows.

1) Divide the time series into two parts: a fitting set $x_{1}, \ldots, x_{N}$ and an observed set $x_{N+1}, \ldots, x_{2 N}$.
2) Choose an embedding dimension $m$, a delay time $\tau$, and a prediction time $T$.

3) Choose a delay vector $\mathbf{X}_{i}=\left(x_{i}, \ldots, x_{i-(m-1) \tau}\right)$ for $i \geq N$.

4) Compute the distance $d_{i j}$ of the test vector $\mathbf{X}_{j}$ from the delay vector $\mathbf{X}_{i}, N-T \geq i \geq 1+(m-1) \tau$, using the maximum norm.

5) Find the $k$ nearest neighbors of $\mathbf{X}_{i(1)}, \ldots, \mathbf{X}_{i(k)}$ of $\mathbf{X}_{j}$ and fit an affine model, where the parameters $\alpha_{0}, \ldots, \alpha_{m}$ are computed by least squares.

6) Repeat step 1)-5) for all $i$ to estimate a $T$ step-ahead prediction value.

7) Compute the linear correlation coefficient $\gamma$ between the observed set and the predicted set.

If the correlation coefficient between the predicted and observed values falls as the prediction extends further into the future, this time series is thus regarded as a chaotic time series. This technique is applicable to any time series showing stationary, periodic, quasi-periodic, or chaotic behavior and can distinguish chaos from noise effectively.

\section{Controlling Mixing Tunneling}

\section{A. Wavepacket}

Asymmetric coupled QW systems of 25-18-49 (first well width-barrier width-second well width in Angstroms) with a barrier height of $0.2506 \mathrm{eV}$ are investigated. In this structure, the $\mathrm{HH}^{a}$ state is aligned with the $\mathrm{LH}^{b}$ state. The Luttinger parameters $\left(\gamma_{1}, \gamma_{2}, \gamma_{3}\right)$ are chosen to be $(6.85,2.1,2.9)$ in the well region and $(5.15,1.39,2.10)$, which are obtained by a linear interpolation of the Luttinger parameters of GaAs and AlAs, in the barrier region. Also, the space interval $\epsilon$ and time interval $\delta$ are chosen to be $1 \AA$ and $1 \mathrm{fs}\left(10^{-15}\right)$. The initial wave functions are the $\mathrm{HH}$ wave functions in the first well, and the tunneling process is initiated at $t=0$.

Mixing tunneling occurs between $\mathrm{HH}$ and $\mathrm{LH}$ states due to band mixing effects. Figs. 2 and 3 show the mixing tunneling in 25-18-49 coupled wells without and with electric field (45 $\mathrm{kV} / \mathrm{cm}$ ), respectively, when $k_{\|}=0.03$. The first and the second well of the coupled QW's is shown by the dotted line at $t=0$ frame. In Fig. $2, \mathrm{HH}^{a}$ to $\mathrm{LH}^{a}$ are significant due to the $k_{\|}$, and $\mathrm{HH}^{a}$ to $\mathrm{LH}^{b}$ are well aligned. Thus, these two processes are compatible. The oscillation of the wavepacket shows no dominant process in either $\mathrm{HH}^{a}$ to $\mathrm{LH}^{a}$ or $\mathrm{HH}^{a}$ to $\mathrm{LH}^{b}$ (The wavepacket appears randomly in the first and the second wells in the LH band). However, in Fig. 3, $\mathrm{HH}^{a}$ to $\mathrm{LH}^{a}$ are significant but $\mathrm{HH}^{a}$ to $\mathrm{LH}^{b}$ becomes misaligned because of the external electric field. Thus, the wavepacket mainly oscillates between $\mathrm{HH}^{a}$ and $\mathrm{LH}^{a}$ states, indicating that the $\mathrm{HH}^{a}$ to $\mathrm{LH}^{a}$ process is dominant.

\section{B. Tunneling Probability}

To characterize the properties of the $\mathrm{HH}$ to $\mathrm{LH}$ mixing tunneling, one can define a mixing tunneling probability $F(t)$ as the probability of finding the $\mathrm{HH}$ in the $\mathrm{HH}$ band

$$
F(t)=\left\langle\phi^{1}(x, t) \mid \phi^{1}(x, t)\right\rangle_{\mathrm{hh}}
$$




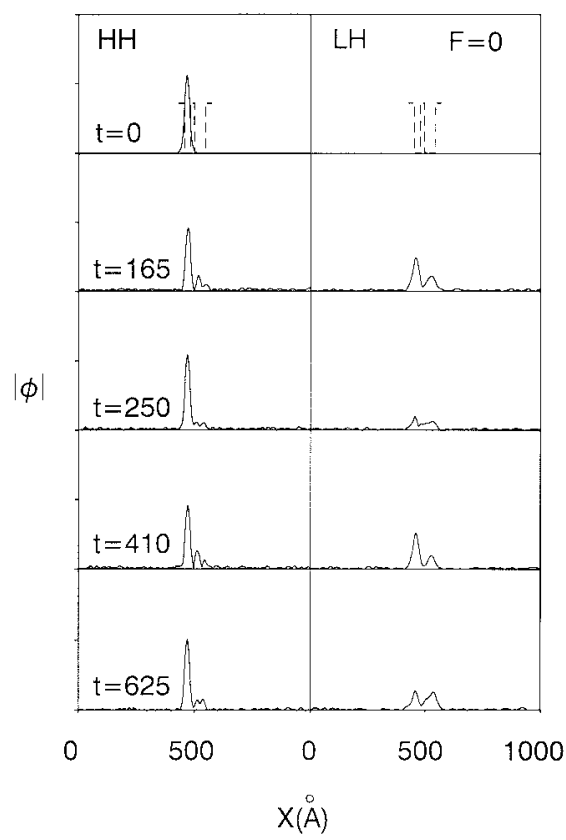

Fig. 2. The mixing tunneling in 25-18-49 coupled wells with zero electric field when $k_{\|}=0.03$. The position of the coupled QW's is shown by the dotted line at $t=0$ frame.

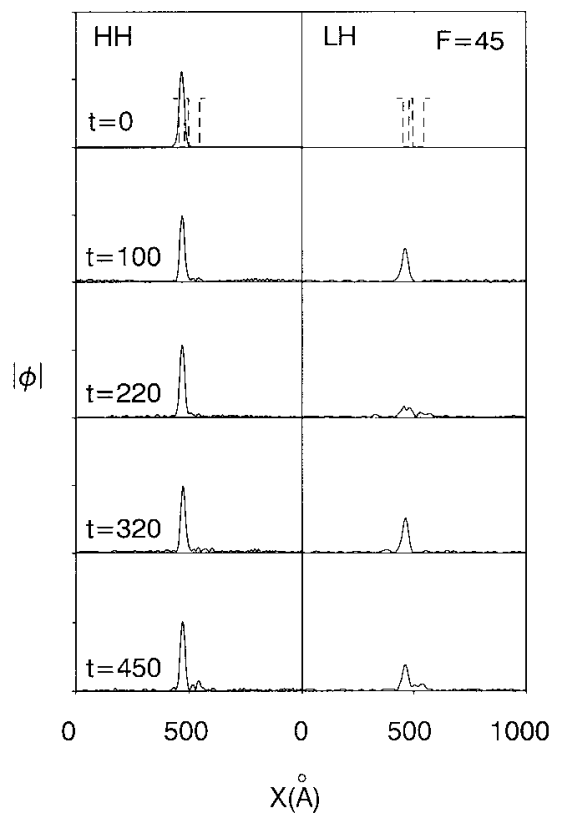

Fig. 3. The mixing tunneling in $25-18-49$ coupled wells with an external electric field of $45 \mathrm{kV} / \mathrm{cm}$ when $k_{\|}=0.03$.

where $\phi^{1}(x, t)$ is the $\mathrm{HH}$ wave function. When $F(t)$ approaches one, the wavepacket is mainly located in the $\mathrm{HH}$ band. When $F(t)$ is small, the wavepacket tunnels into the LH band. By this definition, the nonlinearity is introduced, which is an $L_{2}$ norm resembling the Landau-Lipschitz route to chaos.

Fig. 4 shows the mixing tunneling probability $F(t)$ of the 25-18-49 coupled QW's under various external electric fields $(0,20$, and $45 \mathrm{kV} / \mathrm{cm})$ when $k_{\|}=0.03$. Under zero field, since $\mathrm{HH}^{a}$ to $\mathrm{LH}^{a}$ and $\mathrm{HH}^{a}$ to $\mathrm{LH}^{b}$ are compatible, the

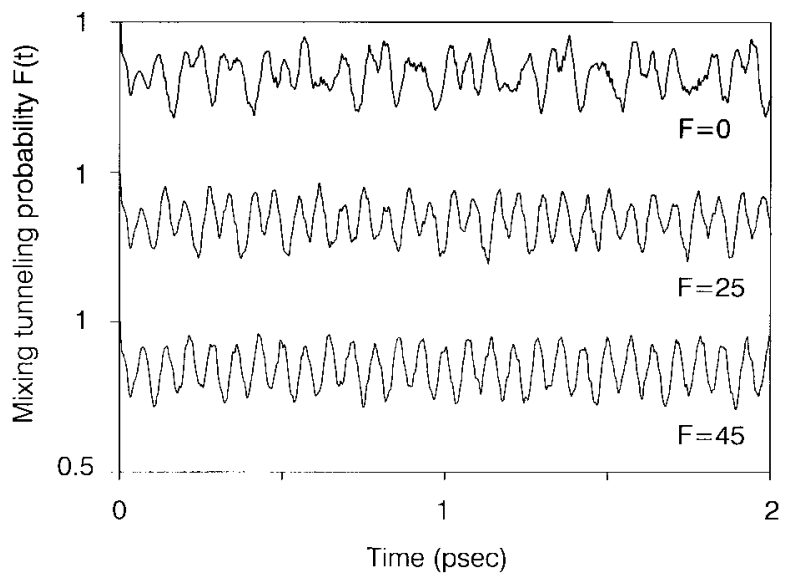

Fig. 4. The mixing tunneling probability $F(t)$ of the 25-18-49 coupled QW's under various external electric fields $(0,20$, and $45 \mathrm{kV} / \mathrm{cm})$ when $k_{\|}=$ 0.03 .

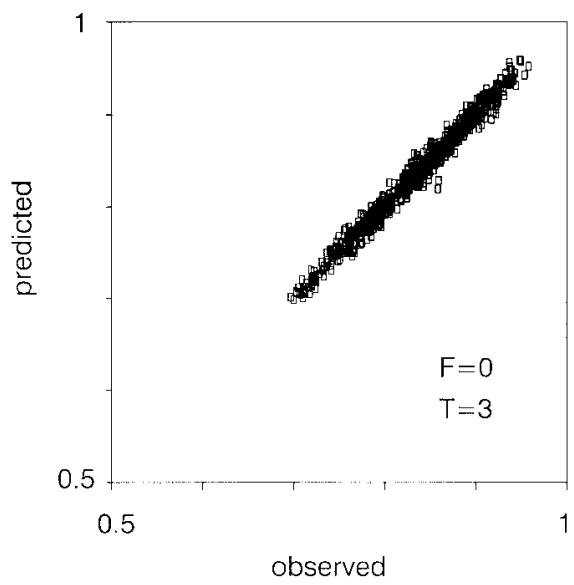

(a)

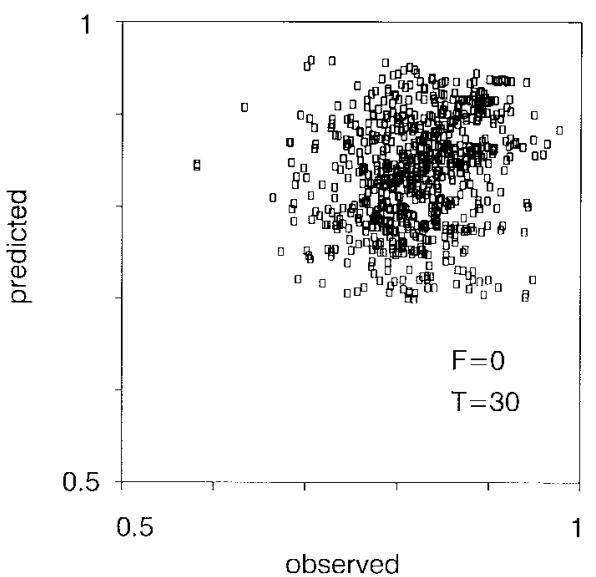

(b)

Fig. 5. Predictions of (a) 3 and (b) 30 time steps into the future versus observed values for $k_{\|}=0.03$ under zero electric field. The first 1000 time points and the second 1000 time points of Fig. 4 are taken to be a fitting set and an observed set $(N=1000)$ with the embedding dimension $m=3$ and delay time $\tau=1$.

mixing tunneling probability $F(t)$ shows much of a disorder, as expected. As the external electric field increases, $\mathrm{HH}^{a}$ and $\mathrm{LH}^{b}$ become misaligned. The $\mathrm{HH}^{a}$ to $\mathrm{LH}^{a}$ process is dominant and $F(t)$ is back to the usual periodic behavior again. 


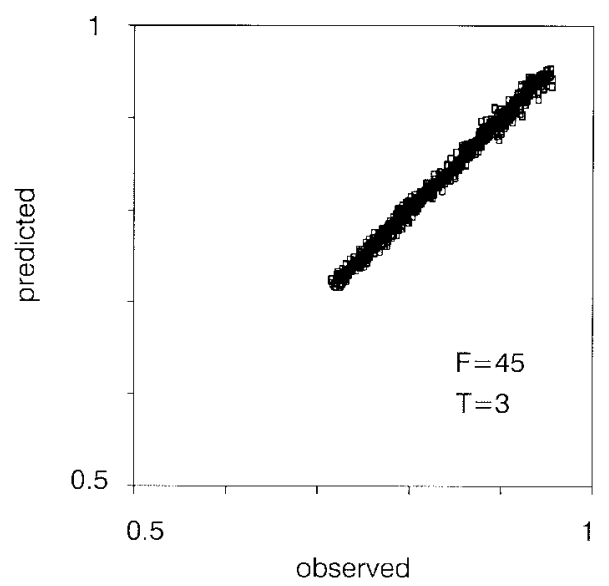

(a)

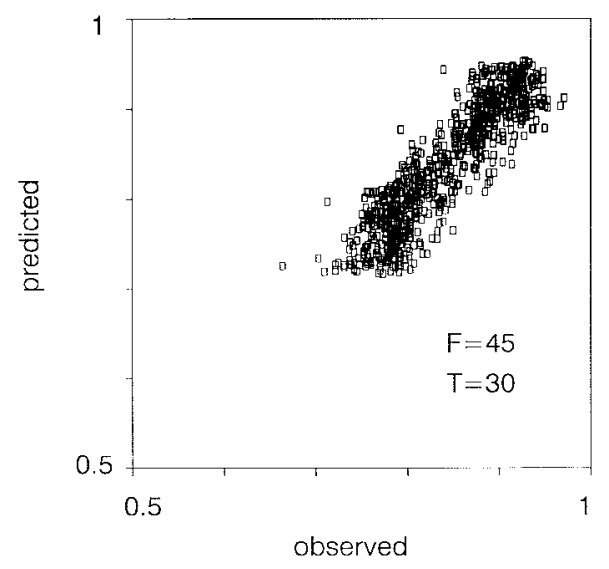

(b)

Fig. 6. Predictions of (a) 3 and (b) 30 time steps into the future versus observed values for $k_{\|}=0.03$ under an external electric field of $45 \mathrm{kV} / \mathrm{cm}$.

\section{Controlling Chaotic Behavior}

To further investigate the inherent properties of $F(t)$, the simplex projection method is used [13], [14]. This method is designed for making short-term and long-term predictions about the trajectories of chaotic dynamical systems. Fig. 5(a) and (b) show predictions of 3 and 30 time steps into the future versus observed values for $k_{\|}=0.03$ under zero field. The first 1000 time points and the second 1000 time points of Fig. 4 are taken to be a fitting set and an observed set $(N=1000)$ with the embedding dimension $m=3$ and delay time $\tau=1$. From 3 to 30 time steps, the loss of the prediction power is clearly indicated, which is interpreted as a characteristic feature of chaos. Similarly, Fig. 6(a) and (b) show predictions of 3 and 30 time steps into the future versus observed values for $k_{\|}=$ 0.03 under an external electric field of $45 \mathrm{kV} / \mathrm{cm}$. From 3 to 30 time steps, the prediction power is maintained, which is interpreted as a characteristic feature of periodical plus noise behavior.

Fig. 7 plots the correlation coefficient $\gamma$ as a function of prediction time under various external electric fields $(0,20$, and $45 \mathrm{kV} / \mathrm{cm}$ ) when $k_{\|}=0.03$. Under zero field, the $\gamma$ falls off significantly due to the loss of prediction power. A decrease in the $\gamma$ with increasing prediction time interval is a characteristic

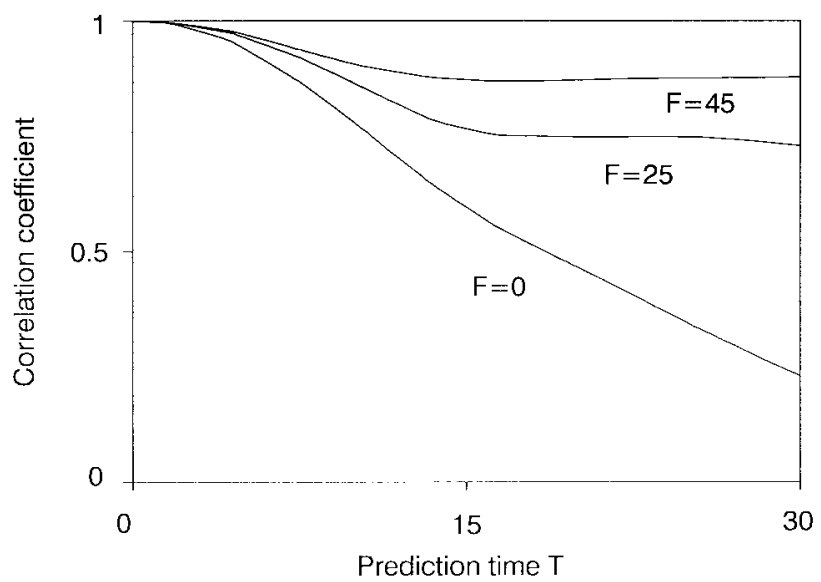

Fig. 7. The correlation coefficient $\gamma$ as a function of prediction time under various external electric fields $(0,20$, and $45 \mathrm{kV} / \mathrm{cm})$ when $k_{\|}=0.03$.

feature of chaos and can also give a rough measure of the Lyapunov exponent. As the external electric field increases, the $\gamma$ changes less, indicating the periodical behavior of $F(t)$ becomes more significant than the chaotic behavior.

To verify the switching between the oscillatory motion and chaotic motion due to the external electric fields, the pump and probe technique, and the time-domain terahertz spectroscopy described in [2], [3], and [9] can be used. Coupled-well samples should be carefully prepared so that the the HH state in the first well is aligned with the LH state in the second well. Note that the alignment between the HH state of the first well and the LH state of the second well can be altered by an appropriate external electric field. Thus, it is expected that chaotic spectroscopy is shown without the electric field, and terahertz oscillatory spectroscopy is shown with the electric field.

\section{CONCLUSION}

The mixing tunneling which occurs between $\mathrm{HH}$ and $\mathrm{LH}$ states can be driven by: 1) the HH to the LH state in the first well due to in-plane vector $k_{\|}$and 2) the HH to the LH state in the second well due to the alignment of the HH state in the first well and the LH state in the second well. If these two tunneling processes are compatible, chaotic behavior occurs. With an external electric field, process 2) becomes dominant and oscillatory is recovered.

\section{REFERENCES}

[1] S. Luryi, "Possibility of a direct observation of the time evolution in heterostructure barrier tunneling," Solid State Commun., vol. 65, pp. 787-789, 1988.

[2] K. Leo, J. Shah, E. O. Göbel, T. C. Damen, S. Schmitt-Rink, and W. Schäfer, "Coherent oscillations of a wave packet in a semiconductor double-quantum-well structures," Phys. Rev. Lett., vol. 66, pp. 201-204, 1991.

[3] H. G. Roskos, M. C. Nuss, J. Shah, K. Leo, D. A. B. Miller, A. M Fox, S. Schmitt-Rink, and K. Köhler, "Coherent submillimeter-wave emission from charge oscillation in a double-well potential," Phys. Rev. Lett., vol. 68, pp. 2216-2219, 1992.

[4] C. Juang, "Interwell coherent tunneling in coupled quantum wells," Phys. Rev. B, vol. 44, pp. 10706-10711, 1991.

[5] V. Sankaran and J. Singh, "Formalism for tunneling of mixed-symmetry electronic states: Application to electron and hole tunneling in direct- 
and indirect-band-gap GaAs/ $\mathrm{Al}_{x} \mathrm{Ga}_{1-x}$ As structures," Phys. Rev. B, vol. 44, pp. 3175-3186, 1991 .

[6] C. Juang, P. A. Chen, and C. Y. Chang, "Valence band mixing effects on hole oscillations in coupled quantum wells," Phys. Rev. B, vol. 47, pp. 4563-4568, 1993.

[7] K. Leo, J. Shah, J. P. Gordon, T. C. Damen, D. A. B. Miller, C. W. Tu, and J. E. Cunningham, "Effect of collisions and relaxation on coherent resonant tunneling: Hole tunneling in $\mathrm{GaAs} / \mathrm{Al}_{x} \mathrm{Ga}_{1-x} \mathrm{As}$ double-quantum-well structures," Phys. Rev. B, vol. 42, pp. 7065-7068, 1990.

[8] T. B. Norris, N. Vodjdani, B. Vinter, E. Costard, and E. Böckenhoff, "Resonant tunneling between heavy-hole and light-hole states in coupled quantum wells," Phys. Rev. B, vol. 43, pp. 1867-1870, 1991.

[9] P. C. M. Planken, M. C. Nuss, I. Brener, K. W. Gossen, M. S. C. Luo, S L. Chuang, and L. Pfeiffer, "Terahertz emission in single quantum wells after coherent optical excitation of light hole and heavy hole excitons," Phys. Rev. Lett., vol. 69, pp. 3800-3903, 1992.

[10] W. A. Lin and L. E. Ballentine, "Quantum tunneling and chaos in a driven anharmonic oscillator," Phys. Rev. Lett., vol. 65, pp. 2927-2930, 1990.

[11] R. Roncaglia, L. Bonci, F. M. Izrailev, B. J. West, and P. Grigolini, "Tunneling versus chaos in the kicked Harper model," Phys. Rev. Lett., vol. 73, pp. 802-805, 1995.

[12] C. Juang, C. B. Tsai, and J. Juang, "Chaotic behavior of hole mixing tunneling in asymmetric coupled quantum wells," Solid State Commun., vol. 101, no. 8, pp. 581-584, 1997.

[13] G. Sugihara and R. M. May, "Nonlinear forecasting as a way of distinguishing chaos from measurement error in time series," Nature, vol. 344, pp. 734-741, 1990.

[14] M. Casdagli, "Chaos and deterministic versus stochastic nonlinear modeling," J. R. Statist. Soc. B, vol. 54, pp. 303-328, 1991.

[15] S. L. Chuang, "Efficient band-structure calculations of strained quantum wells," Phys. Rev. B, vol. 43, pp. 9649-9661, 1991.

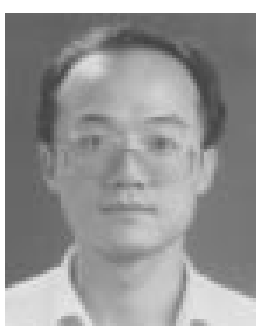

Cheng Juang (M'92) received the B.S. degree in electronic engineering from National Chiao Tung University in 1981, the M.S. degree in electrical engineering from the University of Massachusetts, Amherst, in 1986, and the Ph.D. degree in electrical engineering from the University of Washington, Seattle, in 1990.

After graduation, he joined the Optoelectronics Laboratory, ITRI, in Hsinchu, Taiwan, as a Research Scientist. In 1993, he became a faculty member of the Electronics Department of the Ming Hsin Institute, Taiwan. He is presently an Associate Professor and serves as the department chair. His research interests include nonlinear effects of laser diodes and quantum-well structures.

J. Y. Wang, photograph and biography not available at the time of publication.

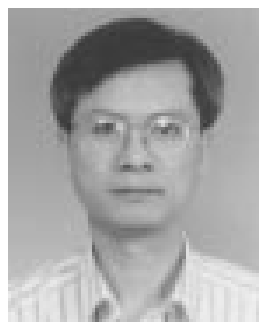

Jonq Juang received the Ph.D. degree in mathematics from Texas Tech University, Lubbock, in 1987.

He held a visiting position at Wright State University, Dayton, OH, from 1987 to 1989 . He has been with National Chiao Tung University, Taiwan, since 1989. In 1995, he was promoted to Ful Professor. His current interests are lattice dynamical and Hamiltonian systems and chaos. 